International Journal of Modern Physics E (c) World Scientific Publishing Company

\title{
Recent progress in the study of fission barriers in covariant density functional theory
}

\author{
A. V. Afanasjev*, H. Abusara \\ Department of Physics and Astronomy, Mississippi State University, Mississippi 39762 USA \\ P. Ring \\ Physik-Department der Technischen Universität München D-85748 Garching, Germany \\ Received (received date) \\ Revised (revised date)
}

Recent progress in the study of fission barriers of actinides and superheavy nuclei within covariant density functional theory is overviewed.

\section{Introduction}

A study of the fission barrier heights $B_{f}^{s t}$ of nuclei is motivated by the importance of this quantity for several physical phenomena. For example, many heavy nuclei decay by spontaneous fission, and the size of the fission barrier is a measure for the stability of a nucleus reflected in the spontaneous fission lifetimes of these nucle: 1 . The $r$-process of stellar nucleosynthesis depends (among other quantities such as masses and $\beta$-decay rates) on the fission barriers of very neutron-rich nuclei2 23 . In addition, the population and survival of hyperdeformed states at high spin also depends on the fission barriers 45 .

The physics of fission barriers is also intimately connected with on-going search for new superheavy elements which is motivated by the attempts to provide the answers for two open questions in nuclear structure, namely, the limits of the existence of atomic nuclei at large values of proton number and the location of the island of stability of superheavy nuclei and the next magic numbers (if any) beyond $Z=82$ and $N=126$. For example, the probability for the formation of a superheavy nucleus in a heavy-ion-fusion reaction is directly connected to the height of its fission barrier ${ }^{6}$ which is a decisive quantity in the competition between neutron evaporation and fission of a compound nucleus in the process of its cooling. The large sensitivity of the cross section $\sigma$ for the synthesis of the fissioning nuclei on the barrier height $B_{f}^{s t}$ also stresses a need for accurate calculations of this value.

*Based on talk presented at 18th Nuclear Physics Workshop "Maria and Pierre Curie", 2011, Kazimierz, Poland 


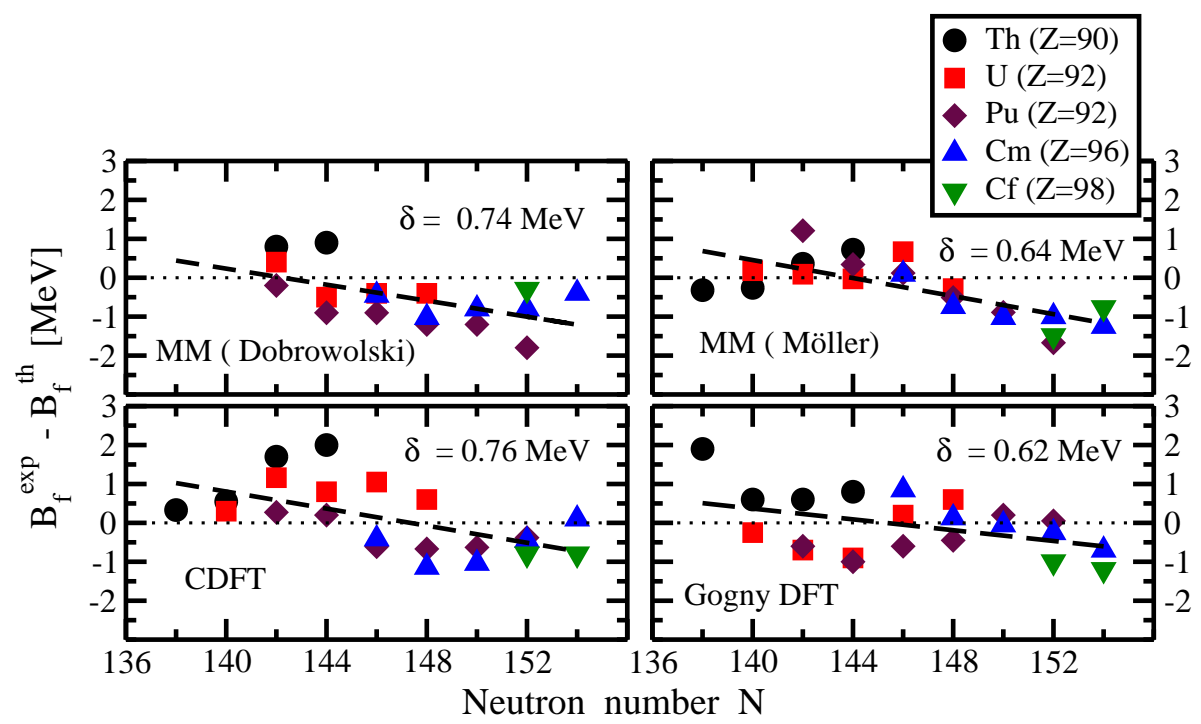

Fig. 1. The difference between experimental and calculated heights of inner fission barriers as a function of neutron number $N$. The results of the calculations are compared to estimated fission barrier heights given in the RIPL-2 database 18, which is used for this purpose in the absolute majority of theoretical studies on fission barriers in actinides. The results of the calculations within microscopic+macroscopic method ('MM(Dobrowolski), 15 and 'MM(Möller)'16), covariant density functional theory ('CDFT' 10 and density functional theory based on the finite range Gogny force ('Gogny DFT' 17) are shown. Thick dashed lines are used to show the average trend of the deviations between theory and experiment as a function of neutron number. The average deviation per barrier $\delta$ [in MeV] is defined as $\delta=\sum_{i=1}^{N}\left|B_{f}^{i}(t h)-B_{f}^{i}(\exp )\right| / N$, where $N$ is the number of the barriers with known experimental heights, and $B_{f}^{i}(t h)\left(B_{f}^{i}(\exp )\right)$ are calculated (experimental) heights of the barriers. Long-dashed lines represent the trend of the deviations between theory and experiment as a function of neutron number. They are obtained via linear regression based on a least square fit.

Covariant density functional theory $(\mathrm{CDFT})^{7}$ provides a natural incorporation of spin degrees of freedom and an accurate description of spin-orbit splittings, which has an essential influence on the underlying shell structure. Lorentz covariance of the CDFT equations leads to the fact that time-odd mean fields are determined with the same constants as time-even fields 8 . In addition, pseudo-spin symmetry finds a natural explanation in the relativistic framework 9 . As a result, CDFT provides an attractive framework for the description of the structure of nuclei. Over the years a large variety of nuclear phenomena have been successfully described within CDFT (see Ref. 7 and references therein). However, until recently most of the calculations were restricted to axial symmetry and therefore it was not possible to provide an accurate description of fission barriers. Only the inclusion of triaxiality in Ref. ${ }^{10}$ has resolved this puzzle. This manuscript will review recent progress in understanding the fission barriers in CDFT. 


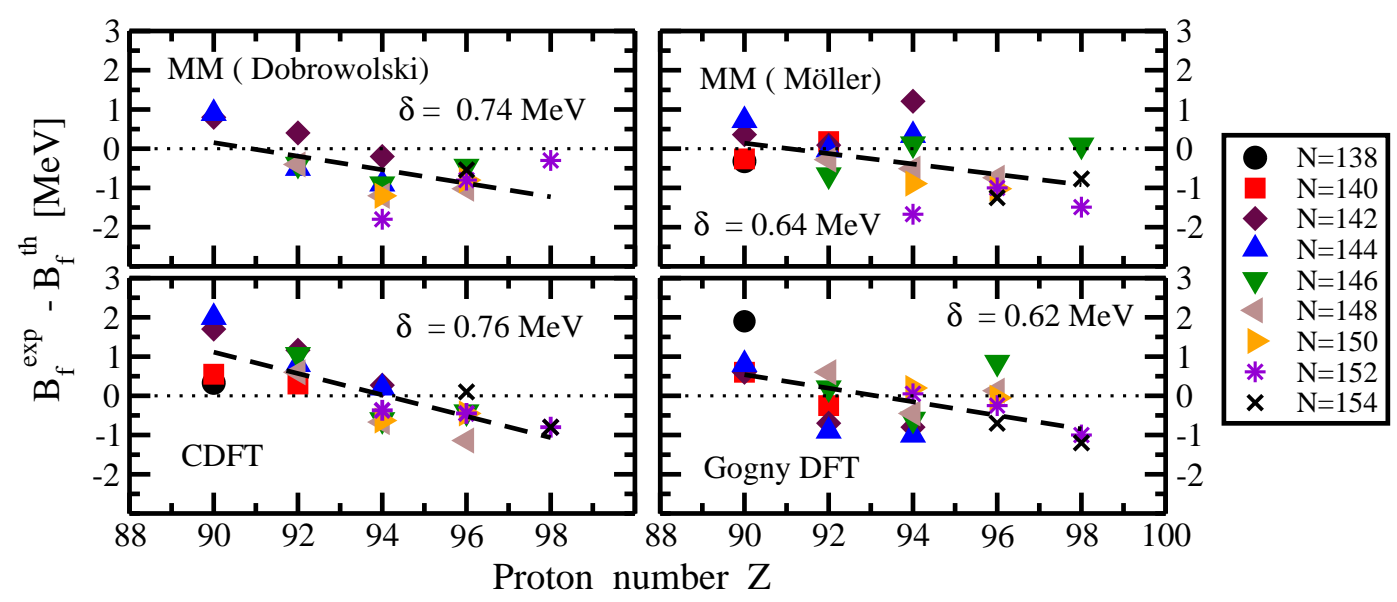

Fig. 2. The same as in Fig. 1 but as a function of proton number $Z$.

\section{Fission barriers in actinides}

Earlier calculations performed within axially symmetric relativistic mean field $(\mathrm{RMF})+\mathrm{BCS}^{11}$ and relativistic Hartree-Bogoliubov (RHB) ${ }^{12}$ frameworks have shown substantial deviations from experiment for the heights of the inner fission barriers. It is known from non-relativistic calculations that triaxiality lowers the saddle in actinides and brings the results of calculations closer to experiment (see discussion in Ref! 10 ). Although a triaxial RHB computer code with finite range Gogny forces in the pairing channel was available for more than 10 years 13 , systematic calculations of fission barriers within this framework are numerically too expensive. This is because a large basis including full $N_{F}=20$ fermionic and $N_{B}=20$ bosonic shells as well as 24 mesh points for the numerical Gauss-Hermite integration are needed for the required accuracy 10 . This problem has been resolved in Ref. ${ }^{10}$ by performing calculations within triaxial RMF+BCS model employing monopole pairing forces instead of full RHB calculations. These simplifications decreased the computational time by at least of one order of magnitude, thus making systematic calculations of inner fission barriers feasible.

The inclusion of triaxiality has improved the accuracy of the description of inner fission barriers in CDFT 10 bringing it up to the level of the accuracy typical for microscopic + macroscopic (MM) approaches and DFT with Gogny forces. Since the results of this investigation performed with the NL3* parametrization of the RMF Lagrangian 14 are already published 10 we will concentrate in this section on the comparative analysis of the results obtained in different theoretical frameworks.

Figs. 1 and 2 show the differences between experimental and calculated heights of inner fission barriers obtained in different theoretical models as a function of neutron and proton numbers, respectively. Note that this comparison covers only results of systematic triaxial calculations of even-even $\mathrm{Th}, \mathrm{U}, \mathrm{Pu}, \mathrm{Cm}$ and $\mathrm{Cf}$ nuclei. To our knowledge, no such calculations have been published with DFT based on 


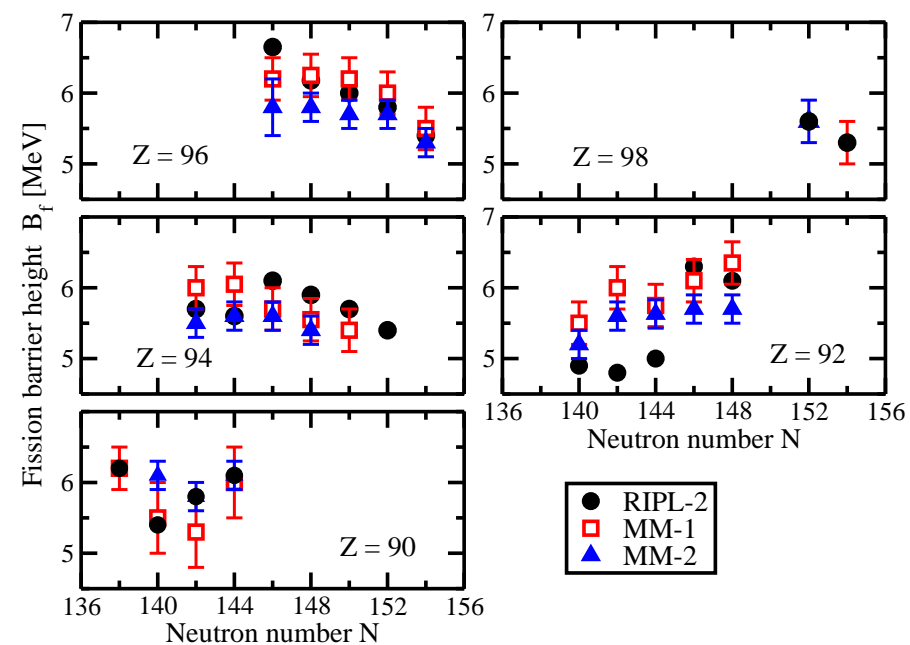

Fig. 3. The heights $\mathrm{B}_{f}$ of inner fission barriers of actinides. The results of the RIPL- 218 , 'MM-1' and 'MM-2 20 compilations are shown. Note that 'MM-1' is based on Refs 2122 , while 'MM-2' on $\operatorname{Ref} \frac{23}{\text {. }}$

Skyrme forces. As a result, these figures cover all existing systematic triaxial studies of inner fission barriers in actinides.

The $\delta$-values displayed on the panels of Figs. 1 and 2 show the average deviation from experiment for the calculated heights of inner fission barriers. One can see that they are of the same magnitude in the different approaches and minor differences between the approaches in the $\delta$-values are not important considering the considerable uncertainties in the extraction of inner fission barrier heights from experimental data (see Fig. 3).

However, the similarity of the average trends of these deviations (shown by thick dashed lines in Figs. (1)and 2) as a function of neutron and proton numbers is more important considering the differences in underlying mean fields and in the treatment of pairing correlations. At present, it is difficult to find a clear explanation for these trends. Although differences in the treatment of pairing correlations (BCS with monopole pairing and of different pairing windows in the $\mathrm{CDFT}^{10}$ and $\mathrm{MM}^{15} 16$ calculations versus the Hartree-Fock-Bogoliubov framework based on the D1S force in Gogny DFT ${ }^{17}$ ) can contribute to deviations between theory and experiment ${ }^{12}$, it is quite unlikely that they are responsible for the observed trends of the deviations.

\section{The impact of the accuracy of the description of the single-particle energies on fission barriers}

An essential difference between the phenomenological models based on WoodsSaxon or Nilsson potentials and self-consistient DFT calculations is the fact that the phenomenological potentials are fitted to experimental single-particle energies while, apart from the adjustment of the spin-orbit force in the Gogny functional, no 
single particle information is used in the fit of the DFT parametrizations. As a consequence, the phenomenological potentials accurately describe the single-particle spectra in deformed systems. On the contrary, several restricted in scope investigations of experimental spectra in deformed odd nuclei24|25|26 showed that many of the DFT models do not possess the same spectroscopic quality for the description of single-particle spectra achievable in the MM method. A recent systematic investigation of the spectra of rare-earth nuclei and actinides in CDFT ${ }^{27}$ strongly enforces this conclusion.

A statistical analysis 27 of discrepancies between calculated and experimental energies of one-quasiparticle states in the ground state minimum is presented in the left panel of Fig. 4. One can see that in the actinide region only approximately $33 \%$ of one-quasiparticle states are described with an accuracy better than $200 \mathrm{keV}$, and approximately $22 \%$ with an accuracy between 200 and $400 \mathrm{keV}$ in the NL3* and NL1 parametrizations of the RMF Lagrangian. The percentage of states for a given range of deviations gradually decreases with increasing deviation between experiment and calculations. However, for some states the deviation of the calculated energy from experiment exceeds $1 \mathrm{MeV}$ and can be close to $1.4 \mathrm{MeV}$. Fig. 4 also shows that with the NL1 parametrization the 1-qp energies in odd-proton rare-earth nuclei are somewhat better described as compared with actinide region. Otherwise, the distribution histograms for the deviations are similar in both regions and for both parametrizations.

In the light of these results, it is important to understand why MM and DFT models describe the experimental fission barriers with similar accuracy (Sect. 2).

First, it is important to remember that theoretical single-particle energies can be confronted with experiment only at the ground state since reliable experimental data on the energies of dominant single-particle states either at the saddle or at the second (superdeformed) minimum are absent despite the fact that fission isomers in actinides have been observed almost 50 years agd. Thus, both types of models (MM and DFT) rely in the study of fission barriers on the "extrapolations" of the energies of single-particle states to large deformations. Since the quality of such "extrapolations" is not known one cannot say a priori that the description of the single-particle energies in MM models is better than in DFT.

Second, there exist important differences between the MM models and DFT in the interpretation of the meaning of theoretical single-particle states and their energies and neither of these interpretations takes fully into account the complicated physics of single-particle degrees of freedom.

It is well known that experimental "single-particle" states are not mean-field states. In reality, their wave functions are fragmented and always contain the admixtures from vibrational phonons. In odd mass nuclei, the weights of these admixtures increase with increasing excitation energy of the level relative to the ground state 28 . By fitting the parameters of phenomenological potentials to the energies

a This is also true for all regions of superdeformation studied so far in experiment. 


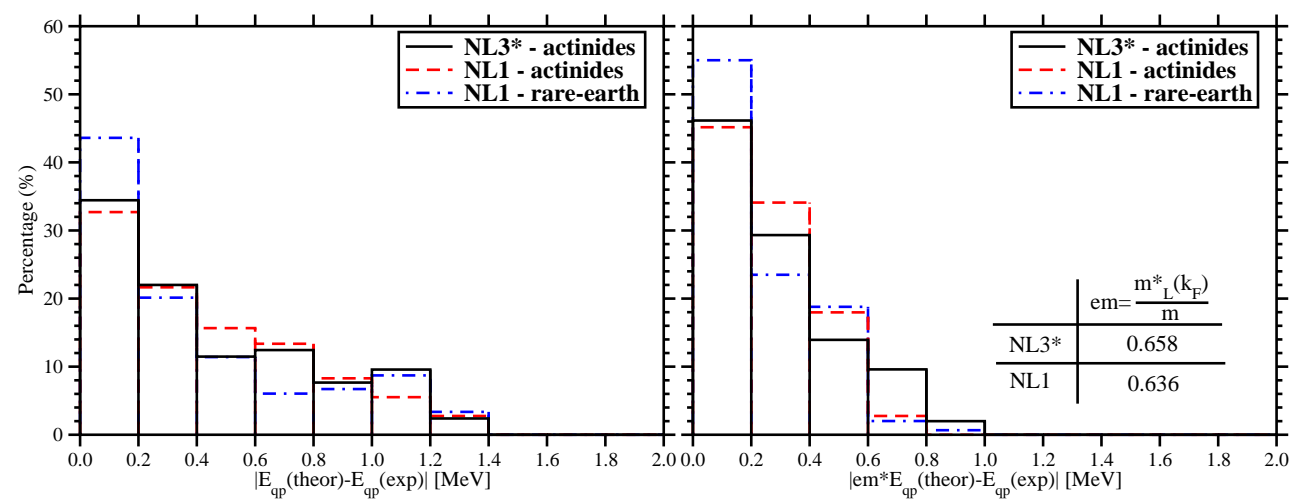

Fig. 4. (left panel) The distribution of the deviations of the calculated energies $E_{q p}($ theor $)$ of one-quasiparticle states from experimental ones $E_{q p}(\exp )$. The vertical axis shows the percentage of the states which deviate from experiment by the energy deviation range (the width of bar) specified on horizontal axis. (right panel) The same as in left panel, but for the case when the energy scale of theoretical spectra is corrected for low Lorentz effective mass.

of dominant single-particle states, the MM models effectively include vibrational corrections into these potentials but only on the level of the energies and not on the level of the wavefunctions. As a consequence, these potentials are characterized by an effective mass of the nucleon at the Fermi level $m^{*}\left(k_{F}\right) / m \approx 1.0$ which reproduces a calculated level density close to experiment.

On the contrary, in density functional methods single-particle levels are not adjusted to experiment since their parameters are fitted mainly to bulk and neutron matter properties. As a consequence, most of them, in particular Gogny and relativistic functionals, are characterized by low effective mass of the nucleon (the Lorentz mass for the case of CDFT), and calculated single-particle states do not include vibrational corrections. A low effective mass leads to a stretching of the theoretical single-particle energy scale as compared with experiment, and, thus, to a larger deviations between theory and experiment for deformed one-quasiparticle states (left panel of Fig. (4). To cure this problem one should go beyond the mean field approximation and supplement CDFT by particle-vibrational coupling $(\mathrm{PVC})^{29}$. So far, this has been done only in spherical nuclei in Refs. 30131, in which it was shown that in the presence of PVC (i) calculated spectra of dominant singleparticle states come closer to experimental ones and (ii) effective mass of the nucleon comes closer to 1 .

A similar compression of calculated spectra is expected also in deformed nuclei. However, so far, no PVC model based on the DFT framework has been developed for such nuclei. The analysis of Ref.27 suggests that on average the expected compression of single-particle spectra can be achieved via a rescaling of one-quasiparticle (1-qp) energies by the Lorentz effective mass. The impact of such an energy rescaling on the distribution of the deviations between theory and experiment is shown

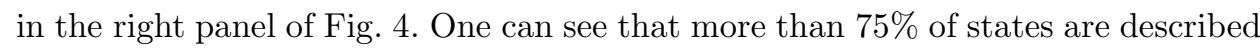


with an accuracy better than $400 \mathrm{keV}$; this is a typical accuracy of the description of the energies of deformed 1-qp states within phenomenological potentials 32 133. Although this energy rescaling is somewhat schematic and assumes that the effect of PVC is identical in spherical and deformed nuclei, it clearly indicates that PVC, leading to an increase of the effective mass, will also improve the description of experimental spectra as compared with mean field results.

\section{Extrapolation to superheavy nuclei}

Systematic CDFT calculations of fission barriers allowing for triaxial deformation are performed for even-even superheavy nuclei with charge numbers $Z=112-120$ and $N-Z=48-62$ using three classes of models 34 . These are nonlinear mesonnucleon coupling, the density-dependent meson-nucleon coupling and densitydependent point coupling models. The main differences between them lay in the treatment of the range of the interaction, the mesons and in the density dependence. The interaction in the first two classes has a finite range, while the third class uses zero-range interaction with an additional gradient term in the scalar isoscalar channel. Mesons are absent in the density-dependent point coupling model. It turns out that the results obtained with these models are similar, so they will be discussed on the example of the nonlinear meson-nucleon coupling model represented by the NL3* parametrization.

The calculations show that inner fission barriers of the $\mathrm{Z}=112$ and 114 isotopes are axially symmetric. In the $\mathrm{Z}=116$ isotopes, the triaxiality has an impact on inner fission barriers of nuclei with $N \geq 178$. The role of triaxiality becomes even more pronounced in the $\mathrm{Z}=118$ and 120 isotopes, especially the ones with neutron number close to $N=184$.

The situation is illustrated in Fig. 5 . There are two fission pathes in the doubly magic nucleus ${ }^{292} 120$. The first fission path shown by a red dashed line starts at spherical shape and then proceeds between two triaxial hills located at moderate deformations $\left(\beta_{2} \sim 0.35, \gamma \sim \pm 30^{\circ}\right)$ and bypasses the axial hill at $\beta_{2} \sim 0.75$ via a $\gamma \sim 7^{\circ}$ path. The $\gamma$-softness of the PES, which exists between the two triaxial hills, has only a minor effect on the shoulder of the inner fission barrier; however, the height of inner fission barrier is not affected by triaxiality. Second fission path shown by a solid red line starts at spherical shape, and proceeds along the axially symmetric $\gamma=60^{\circ}$ axis, via a saddle point at $\left(\beta_{2} \sim 0.3, \gamma \sim 27^{\circ}\right)$ and then along the first fission path after second minimum. The unusual physical feature of this second path is the fact that initially the nucleus has to be squeezed along the axis of symmetry, thus creating an oblate deformation with $\beta_{2} \sim 0.35$. This is contrary to the usual picture of fission were the nucleus is stretched out along the axis of symmetry having a prolate deformation. A similar fission path also exists in the ${ }^{304} 120$ nucleus. However, the first fission path in this nucleus is modified due to the emergence of a axial hill at $\beta_{2} \sim 0.2$; this shifts the saddle point from $\left(\beta_{2} \sim 0.35, \gamma=0^{\circ}\right)$ in the ${ }^{292} 120$ nucleus to $\left(\beta_{2} \sim 0.30, \gamma \sim 27^{\circ}\right)$ in the ${ }^{304} 120$ 
nucleus.

It is clear that the landscape of the PES of these nuclei in the region of the first fission barrier is more complicated than in the case of the actinides. This calls for finding the dynamical path along which the fission process takes place. Note that non-relativistic calculations based on the MM approach $\frac{35}{35}$ showed that although triaxiality lowers the static fission barriers, it plays a minor role in spontaneous fission of superheavy nuclei with $Z \leq 120$. This is because a fission path via an oblate shape and triaxial saddles is substantially longer as compared with axially symmetric path which leads to significant reduction of penetration probability.

Note that contrary to actinide nuclei, the triaxiality has a considerable impact on the shapes and the heights of outer fission barriers of superheavy nuclei; the later are lowered by $\sim 2-3 \mathrm{MeV}$.
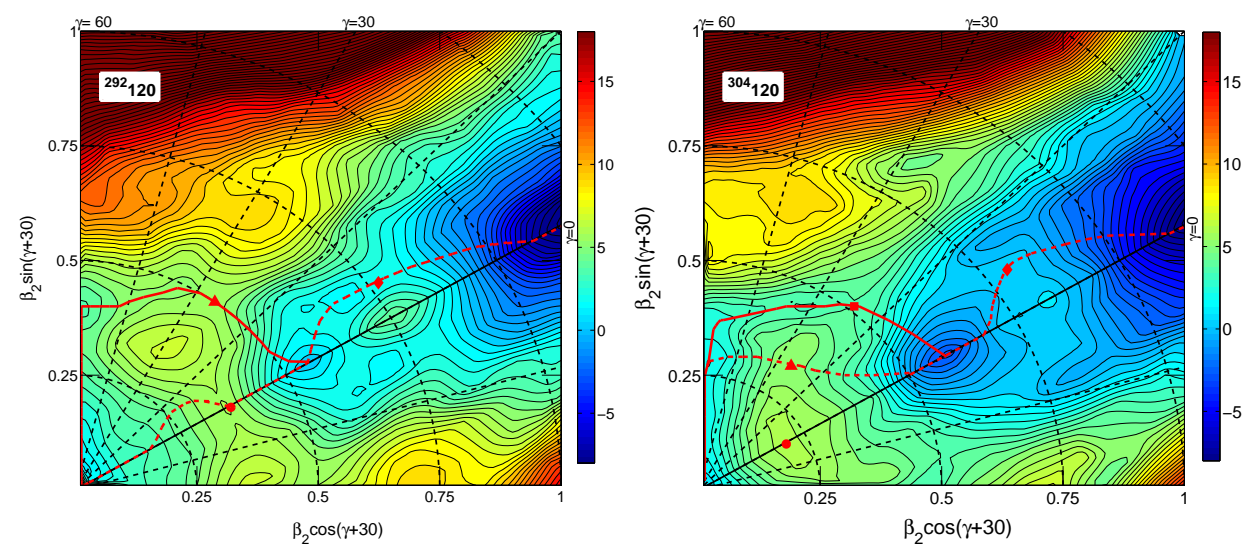

Fig. 5. Potential energy surfaces of the $Z=120, N=172$ (left panel) and $Z=120, N=184$ (right panel) nuclei. The energy difference between two neighboring equipotential lines is equal to 0.5 $\mathrm{MeV}$. The saddles are shown by solid symbols. The saddles are defined via the immersion method (Ref. 16), while the fission path as a minimum energy path which represents the most probable pathway connecting two minima via a given saddle (see, for example, Ref. ${ }^{36}$ ).

\section{Conclusions}

In conclusion, the level of accuracy of the description of fission barriers in actinides is comparable for the methods discussed here, the macroscopic+microscopic method, the covariant and Gogny density functional theories. Similar trends for the deviations from experiment as a function of particle number seen in these approaches may indicate that further substantial improvement can be achieved only in models which go beyond mean field. The impact of the accuracy of the description of the single-particle spectra in different models on the accuracy of reproduction of fission barriers has also been discussed. It was shown that the landscape of the potential energy surface in some superheavy nuclei is more complicated than in the 
case of actinides. This indicates the need for more investigations to determine the dynamical fission path in these nuclei.

This work has been supported by the U.S. Department of Energy under the grant DE-FG02-07ER41459 and by the DFG cluster of excellence "Origin and Structure of the Universe" (www.universe-cluster.de).

\section{References}

1. A. Sobiczewski and K. Pomorski, Prog. Part. Nucl. Phys. 58, 292 (2007).

2. M. Arnould and K. Takahashi, Rep. Prog. Phys. 62, 395 (1999).

3. A. Mamdouh, J. M. Pearson, M. Rayet, and F. Tondeur, Nucl. Phys. A679, 337 (2001).

4. J. Dudek, K. Pomorski, N. Schunck, and N. Dubray, Eur. Phys. J. A20, 15 (2004).

5. A. V. Afanasjev and H. Abusara, Phys. Rev. C 78, 014315 (2008).

6. M. G. Itkis, Y. T. Oganessian, and V. I. Zagrebaev, Phys. Rev. C 65, 044602 (2002).

7. D. Vretenar, A. V. Afanasjev, G. A. Lalazissis, and P. Ring, Phys. Rep. 409, 101 (2005).

8. A. V. Afanasjev and H. Abusara, Phys. Rev. C 81, 014309 (2010).

9. J. N. Ginocchio, Phys. Rev. Lett. 78, 436 (1997).

10. H. Abusara, A. V. Afanasjev, and P. Ring, Phys. Rev. C 82 (2010) 044303.

11. T. Bürvenich, M. Bender, J. A. Maruhn, and P.-G. Reinhard, Phys. Rev. C 69, 014307 (2004).

12. S. Karatzikos, A. V. Afanasjev, G. A. Lalazissis, and P. Ring, Phys. Lett. B689, 72 (2010).

13. A. V. Afanasjev, P. Ring, and J. König, Nucl. Phys. A676, 196 (2000).

14. G. A. Lalazissis, S. Karatzikos, R. Fossion, D. Peña Arteaga, A. V. Afanasjev, and P. Ring, Phys. Lett. B671, 36 (2009).

15. J. Dobrowolski, K. Pomorski, and J. Bartel, Phys. Rev. C 75, 024613 (2007).

16. P. Möller, A. J. Sierk, T. Ichikawa, A. Iwamoto, R. Bengtsson, H. Uhrenholt, and S. Åberg, Phys. Rev. C 79, 064304 (2009).

17. J.-P. Delaroche, M. Girod, H. Goutte, and J. Libert, Nucl. Phys. A771, 103 (2006).

18. RIPL-2 stands for reference input parameter library of International Atomic Energy Agency located at http://www-nds.iaea.org/ripl2/, which for actinides is based on Ref. 19 .

19. V. M. Maslov. RIPL-1 Handbook. TEXDOC-000, IAEA, Vienna, 1998, Ch. 5.

20. D. G. Madland and P. Möller, Los Alamos National Laboratory unclassified report, LA-UR-11-11447 (2011).

21. B. B. Back, O. Hansen, H. C. Britt, and J. D. Garrett, Phys. Rev. C 9, 1924 (1974).

22. H. C. Britt, in Proc. of the Symposium on the Physics and Chemistry of Fission, Jülich, Germany, 14-18 May 1979 (IAEA, Vienna, 1980), Vol. I, p. 3.

23. S. Bjornholm and J. E. Lynn, Rev. Mod. Phys. 52, 725 (1980) and references therein.

24. A. V. Afanasjev et al, Phys. Rev. C 67, 024309 (2003).

25. N. Schunck, J. Dobaczewski, J. McDonnell, J. Moré, W. Nazarewicz, J. Sarich, and M. V. Stoitsov, Phys. Rev. C 81, 024316 (2010).

26. R. Rodriguez-Guzman, P. Sarriguren, and L. M. Robledo, Phys. Rev. C 82, 061302(R) (2010).

27. A. V. Afanasjev and S. Shawaqfeh, Phys. Lett. B706, 177 (2011).

28. V. G. Soloviev, Theory of Atomic Nuclei: Quasiparticles and Phonons (Institute of Physics Publishing, Bristol and Philadelphia, 1992).

29. P. Ring and E. Werner, Nucl. Phys. A211, 198 (1973). 
10 A. V. Afanasjev et. al

30. E. Litvinova and P. Ring, Phys. Rev. C 73, 044328 (2006).

31. E. V. Litvinova and A. V. Afanasjev, Phys. Rev. C 84, 014305 (2011).

32. A. K. Jain, R. K. Sheline, P. C. Sood, and K. Jain, Rev. Mod. Phys. 62, 393 (1990).

33. A. Parkhomenko and A. Sobieczewski, Acta Phys. Polonica 35, 2447 (2004).

34. H. Abusara, A. V. Afanasjev, and P. Ring, submitted to Phys. Rev. C.

35. R. A. Gherghescu, J. Skalski, Z. Patyk, and A. Sobiczewski, Nucl. Phys. A651 (1999) 237.

36. D. Sheppard, R. Tyrell and G. Henkelman, Jour. Chem. Phys. 128 (2008) 134106. 